\title{
REVIEW
}

\section{The effectiveness of physical interventions for people with spinal cord injuries: a systematic review}

\author{
LA Harvey, C-WC Lin, JV Glinsky and A De Wolf \\ Rehabilitation Studies Unit, Northern Clinical School, Faculty of Medicine, University of Sydney, New South Wales, Australia
}

\begin{abstract}
Study design: Systematic review.
Objectives: To provide a quantitative analysis of all randomized controlled trials designed to determine the effectiveness of physical interventions for people with spinal cord injury $(\mathrm{SCl})$.

Setting: Sydney, Australia.

Methods: A search was conducted for randomized controlled trials involving physical interventions for people with $\mathrm{SCl}$. Two reviewers independently rated methodological quality using the PEDro scale and extracted key findings from the trials.

Results: Four thousand five hundred and forty three abstracts were identified of which 31 trials met the inclusion criteria. Trials examined the effectiveness of fitness and strength training $(n=7)$, gait training $(n=5)$, hand therapy $(n=3)$, stretch $(n=4)$, acupuncture $(n=3)$, hand splinting $(n=2)$ and other related therapies $(n=7)$. Six trials reported a between-group mean difference with a clearly important treatment effect on at least one outcome measure. These trials supported the use of fitness, strength and gait training as well as acupuncture.

Conclusion: There is initial evidence supporting the effectiveness of some physical interventions for people with $\mathrm{SCl}$. However, there is a pressing need for high-quality trials to determine the effectiveness of all physical interventions commonly administered in clinical practice.

Spinal Cord (2009) 47, 184-195; doi:10.1038/sc.2008.100; published online 26 August 2008
\end{abstract}

Keywords: rehabilitation; spinal cord injury; systematic review

\section{Introduction}

Many different types of physical interventions are routinely provided to people with spinal cord injury (SCI) as part of their rehabilitation programmes and ongoing care. They include interventions such as strength, fitness and gait training, splinting, stretching and hand therapy. These interventions are typically provided by physiotherapists, occupational therapists, exercise physiologists, medical practitioners and other health-care providers. Physical interventions often target specific impairments such as poor strength, cardiovascular fitness, skill and joint mobility, or impairments related to muscle extensibility, bone loss, pain or spasticity. Each of these impairments impose activity limitations that directly or indirectly prevent patients from performing physical activities such as walking, using their hands, mobilizing in a wheelchair and attending to self-care. Physical interventions that address impairments invariably also address activity limitations. By reducing activity limitations, physical interventions address the ultimate aim of rehabilitation, namely to increase participation and thereby improve overall quality of life.

Correspondence: Dr LA Harvey, Rehabilitation Studies Unit, Royal Rehabilitation Centre Sydney, PO Box 6, Ryde, Sydney, New South Wales 1680, Australia. E-mail: I.harvey@usyd.edu.au

Received 5 May 2008; revised 23 June 2008; accepted 6 July 2008; published online 26 August 2008
Large numbers of physical interventions are advocated for people with SCI. The challenge for clinicians is to ascertain which interventions are most effective. The best evidence for treatment effectiveness comes from high-quality randomized controlled trials. ${ }^{1,2}$ Some of the key strategies important for minimizing bias in randomized controlled trials include concealing allocation, blinding assessors and performing intention-to-treat analyses.

Recent and comprehensive clinical guidelines have synthesized the evidence supporting physical interventions for people with SCI. ${ }^{3,4}$ However, these guidelines do not always determine or interpret the size and associated uncertainty of between-group differences. In addition, they do not include some of the commonly administered physical interventions that have been evaluated within randomized controlled trials. The purpose therefore of this systematic review was to provide a quantitative analysis of all randomized controlled trials designed to determine the effectiveness of physical interventions for people with SCI.

\section{Methods}

Search strategy

The following databases were searched up until December 2007: Medline (from 1966), CINAHL (from 1982), Embase 
(from 1980), the Cochrane Central register of controlled trials and the Physiotherapy Evidence Database (PEDro). ${ }^{5}$ A sensitive search strategy for identifying randomized controlled trials was used ${ }^{6}$ along with the following $\mathrm{MeSH}$ terms: parapleg\$, quadripl\$, tetrapleg\$, wheelchair\$ and spinal cord. This search strategy was adjusted for each database. In addition, the bibliographies of relevant systematic reviews and clinical guidelines were hand searched.

\section{Inclusion criteria}

The inclusion criteria were as follows:

Type of trials: Randomized controlled trials written in English. Crossover trials were included provided allocation to the treatment schedule was randomized. In trials that randomly allocated subjects to experimental groups but included data from non-randomized control conditions, ${ }^{7}$ only data from the randomized groups were included.

Type of participants: Trials in which at least $75 \%$ of participants had sustained a SCI. There were no restrictions on the basis of time since injury, type of injury or age.

Type of interventions: Trials involving the administration of a physical intervention typically provided by health-care professionals. Only trials that involved a treatment administered over more than one occasion were included. In trials that looked at the effects of one-off treatments as well the effects of a series of treatments, ${ }^{8,9}$ only the data reflecting the response to the series of treatments were included. Trials that examined the effectiveness of providing education or equipment were excluded, as were trials directed at respiratory care or skin management.

Type of comparisons: Trials comparing a physical intervention with control (including sham) or no intervention and trials comparing two or more physical interventions.

Types of outcomes: All physical and non-physical outcome measures were included.

\section{Data collection and analysis}

Two reviewers identified potentially eligible trials from the search. Full copies of these trials were attained and again screened for eligibility. Any disagreement between the two reviewers was resolved by a third independent reviewer. If trials were reported in more than one publication, only data from the key publication were included.

The following data were independently extracted by two reviewers: details about the subjects (including classification according to the International Standards for the Classification of SCI, time since injury and number), intervention/s (including type, dosage and duration), outcomes (including type, number and data collection points) and the statistical significance of between-group differences (as stated by trial authors). In all trials, data collected at the beginning and at the end of the intervention period were extracted. The exception was the study by Crowe et al., ${ }^{10}$ where the post-intervention data were collected 6 weeks after the intervention period for all but one outcome. In trials with more than two groups, only data from the two most contrasting groups were extracted.
Between-group mean differences and 95\% confidence intervals (CIs) were extracted for each continuous outcome. ${ }^{11}$ If between-group mean differences and corresponding 95\% CIs were not reported, they were derived from standard deviations, standard errors or $P$-values, provided data were not obviously skewed. ${ }^{12-14}$ Data were extracted from figures, and authors were contacted to clarify ambiguities if necessary and feasible.

The 95\% CI associated with the between-group mean difference for each outcome was used to determine if the effect of the intervention was large enough to be worthwhile. This was done by nominating minimally important between-group differences; the smallest added benefit of an intervention required to justify the time, cost and inconvenience associated with providing the intervention. ${ }^{15,16}$ The minimally important between-group difference was set $a$ priori at $10 \%$ of the combined values of experimental and control groups at the commencement of the trial unless the authors of trials explicitly nominated otherwise. In trials that did not provide initial values, $10 \%$ of the combined values of experimental and control groups at the completion of the trial were used. Results were then categorized in the following way:

(1) Clearly important between-group difference: The 95\% CI associated with the between-group mean difference was larger than the minimally important difference (see Figure 1). ${ }^{15}$

(2) Inconclusive: The 95\% CI associated with the between-group mean difference spanned the minimally important difference (see Figure 1). ${ }^{15}$

(3) Ineffective: The 95\% CI associated with the betweengroup mean difference was smaller than the minimally important difference (see Figure 1). ${ }^{15}$

This categorization takes into account the size and the uncertainty associated with treatment effects and emphasizes clinical significance rather than statistical significance. This methodology appropriately restricts conclusions to the comparisons made in trials. For example, in trials that compare two interventions without a control group, the categorization can only be used to make inferences about the relative effectiveness of the two interventions, not about the effectiveness of one intervention compared to no intervention. Skewed and categorical data were not categorized for clinical significance.

The quality of each trial was independently assessed by two reviewers using the PEDro scale. ${ }^{5}$ Any disagreements were resolved by an independent third person (reviewers did not rate their own trials). The PEDro scale assesses 10 key design features important for minimizing bias and interpreting between-group differences. It rates trials according to whether they did or did not use random allocation; conceal allocation; demonstrate baseline similarity; blind subjects, therapists and assessors; obtain outcome measures from more than $85 \%$ of subjects; provide measures of variability; use intention-to-treat analyses; and perform between-group statistical comparisons. Ratings were based on the written text and not on personal communications. 


\title{
Between-group statistical significance and clearly important
}

Taylor et $\mathrm{al}^{32}$

1: Arm cranking vs

2: No intervention

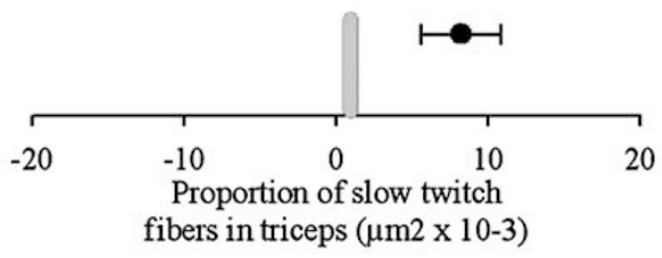

Between-group statistical significance and inconclusive

Ben et $\mathrm{al}^{20}$

1: Weight-bearing standing vs

2: Non weight-bearing standing

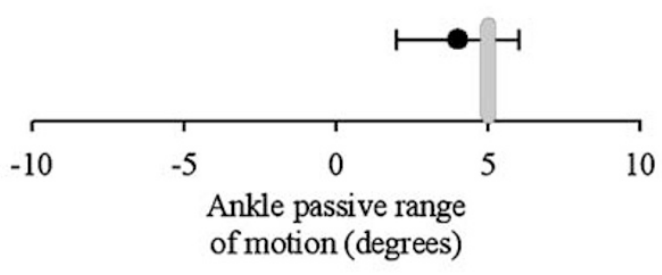

No between-group statistical significance and inconclusive

Dyson-Hudson et $\mathrm{al}^{25}$

1: Acupuncture vs

2: Shame acupuncture

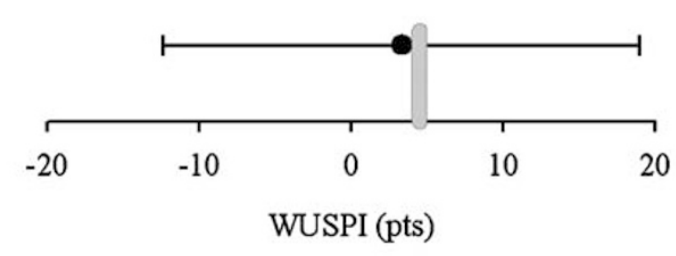

\author{
No between-group statistical significance and \\ ineffective \\ Harvey et $\mathrm{al}^{21}$ \\ 1: Ankle stretch vs \\ 2: No intervention
}

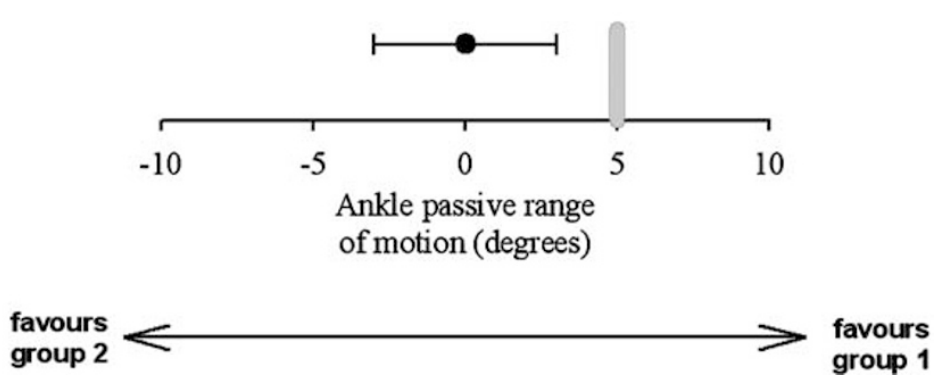

Figure 1 Examples of outcomes from trials demonstrating the four types of results, namely, between-group statistical significance and clearly important treatment effects; between-group statistical significance and inconclusive treatment effects; no between-group statistical significance and inconclusive treatment effects; and no between-group statistical significance and ineffective treatments. The shaded vertical lines indicate the minimally important differences. WUSPI refers to Wheelchair User's Shoulder Pain Index.

\section{Results}

Four thousand five hundred and forty three titles and abstracts were screened. Of these, 65 full papers were retrieved for further screening. Thirty-one trials met the inclusion criteria (see Table 1). The total number of subjects included in all the trials was 770 . Three trials ${ }^{23,29,38}$ had more than 50 subjects. Fifteen trials had 20 or less subjects and the remaining 13 trials had between 20 and 50 subjects. Susceptibility to bias was a problem common to most trials. The median (interquartile range) PEDro score for trials was 4 (3-5; see Table 1$)$. Only 12 trials blinded assessors $^{8,10,23,29,31-33,35-37,40,43}$ and only six trials concealed allocation $^{10,30-33,35}$ and performed intention-to-treat analyses. $^{23,31-33,35,37}$ Dropouts were also a common problem, with only 18 trials reporting outcome data on at least $85 \%$ of subjects. ${ }^{8,9,18-20,22-24,27,28,31-35,37,40,41}$ Not surprisingly and due to the nature of the interventions, only two trials blinded subjects ${ }^{37,43}$ and no trial blinded therapists. 
Table 1 Details of included trials

\begin{tabular}{|c|c|c|c|c|c|c|c|c|}
\hline \multicolumn{4}{|c|}{ Trial details } & \multicolumn{2}{|c|}{ Subjects } & \multicolumn{3}{|c|}{ Between-group differences } \\
\hline Trial & Groups & Dosage & Design & No. & Details & Outcomes & $\begin{array}{l}\text { Statistical } \\
\text { significance }\end{array}$ & Clinical significance \\
\hline \multicolumn{9}{|c|}{ Fitness and strength training } \\
\hline $\begin{array}{l}\text { Baldi et al. }{ }^{17} \\
\text { PEDro }=4\end{array}$ & $\begin{array}{l}\text { 1. ES-driven leg cranking } \\
\text { 2. No intervention }\end{array}$ & $\begin{array}{l}0.5 \mathrm{~h} \\
3 \text { times per week }\end{array}$ & Between-subject & 26 & $\begin{array}{l}\text { Acute } \\
\text { Motor complete }\end{array}$ & Lower limb lean body mass. & SS & Clearly important \\
\hline Blinding $=$ no & $\begin{array}{l}\text { 3. Isometric ES-driven } \\
\text { leg exercises }\end{array}$ & 6 months & & & $\mathrm{C} 5-\mathrm{T} 12$ & $\begin{array}{l}\text { Total lean body mass, } \\
\text { gluteal lean body mass. }\end{array}$ & SS & Inconclusive \\
\hline \multirow[t]{2}{*}{$\begin{array}{l}\text { de Groot et al. }{ }^{18} \\
\text { PEDro }=4 \\
\text { Blinding }=\text { no }\end{array}$} & $\begin{array}{l}\text { 1. High-intensity arm } \\
\text { cranking } \\
\text { 2. Low-intensity arm } \\
\text { cranking }\end{array}$ & $\begin{array}{l}1 \mathrm{~h} \\
3 \text { times per week } \\
8 \text { weeks }\end{array}$ & Between-subject & 6 & $\begin{array}{l}\text { Acute } \\
\text { Mixed } \\
\text { C5-L1 }\end{array}$ & $\begin{array}{l}\mathrm{VO}_{2} \text { peak, insulin sensitivity, } \\
\text { ratio total cholesterol:high- } \\
\text { density lipoprotein cholesterol, } \\
\text { triglycerides. }\end{array}$ & SS & Not categorized \\
\hline & & & & & & $\begin{array}{l}\text { Power output, total cholesterol, } \\
\text { high-density lipoprotein } \\
\text { cholesterol, low-density } \\
\text { lipoprotein cholesterol. }\end{array}$ & No SS & Not categorized \\
\hline \multirow[t]{2}{*}{$\begin{array}{l}\text { Hartkopp et al. } \\
\text { PEDro }=3 \\
\text { Blinding }=\text { no }\end{array}$} & $\begin{array}{l}\text { 1. High-resistance wrist } \\
\text { extensor exercises+ES } \\
\text { 2. Low-resistance wrist }\end{array}$ & $\begin{array}{l}30 \text { min } \\
5 \text { times per week } \\
12 \text { weeks }\end{array}$ & Between-subject & 18 & $\begin{array}{l}\text { Chronic } \\
\text { Not specified } \\
\text { C5/C6 }\end{array}$ & $\begin{array}{l}\text { Fatigue resistance ratio, cost } \\
\text { of contraction. }\end{array}$ & $\begin{array}{l}\text { Not } \\
\text { provided }\end{array}$ & Inconclusive \\
\hline & extensor exercises+ES & & & & & $\begin{array}{l}\text { Peak tension with } 15,30 \text { and } \\
50 \mathrm{~Hz} \text { stimulation, maximal voluntary } \\
\text { contractions. }\end{array}$ & $\begin{array}{l}\text { Not } \\
\text { provided }\end{array}$ & Insufficient data \\
\hline \multirow[t]{3}{*}{$\begin{array}{l}\text { Hicks et al. }{ }^{19} \\
\text { PEDro }=5 \\
\text { Blinding }=\text { no }\end{array}$} & $\begin{array}{l}\text { 1. General strength and } \\
\text { fitness training } \\
\text { 2. Education and }\end{array}$ & $\begin{array}{l}2 \mathrm{~h} \\
2 \text { times per week } \\
9 \text { months }\end{array}$ & Between-subject & 34 & $\begin{array}{l}\text { Chronic } \\
\text { Mixed } \\
\text { C4 and below }\end{array}$ & $\begin{array}{l}\text { Perceived exertion ( } 1 \text { test), perceived } \\
\text { health, pain. }\end{array}$ & SS & Clearly important \\
\hline & relaxation & & & & & $\begin{array}{l}\text { Heart rate ( } 3 \text { tests), power } \\
\text { output ( } 1 \text { test), upper limb } \\
\text { strength ( } 5 \text { muscle groups), } \\
\text { quality of life, stress, satisfaction } \\
\text { with physical function, perceived } \\
\text { exertion ( } 1 \text { test), depression. }\end{array}$ & SS & Inconclusive \\
\hline & & & & & & $\begin{array}{l}\text { Heart rate (resting), power } \\
\text { output ( } 2 \text { tests), blood pressure } \\
\text { (resting), deltoid strength } \\
\text { (left), satisfaction with physical } \\
\text { appearance, perceived } \\
\text { exertion ( } 1 \text { test). }\end{array}$ & No SS & Inconclusive \\
\hline $\begin{array}{l}\text { McLean et al. } .^{20} \\
\text { PEDro }=5\end{array}$ & $\begin{array}{l}\text { 1. Arm cranking } \\
\text { in supine }\end{array}$ & 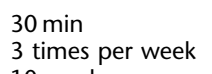 & Between-subject & 15 & $\begin{array}{l}\text { Chronic } \\
\text { Not specified }\end{array}$ & Power (supine and sitting). & SS & Insufficient data \\
\hline \multirow[t]{3}{*}{ Blinding $=$ no } & $\begin{array}{l}\text { 2. Arm cranking } \\
\text { in sitting }\end{array}$ & 10 weeks & & & C5-T1 & Endurance. & No SS & Inconclusive \\
\hline & & & & & & $\begin{array}{l}\mathrm{VO}_{2} \text { peak (supine and sitting), } \\
\text { skinfolds (4 tests), cardiac } \\
\text { output, stroke volume. }\end{array}$ & No SS & Insufficient data \\
\hline & & & & & & $\begin{array}{l}\text { Resting heart rate (sitting and } \\
\text { supine), peak heart rate } \\
\text { (sitting and supine), body } \\
\text { weight, power (sitting). }\end{array}$ & $\begin{array}{l}\text { Not } \\
\text { provided }\end{array}$ & Insufficient data \\
\hline
\end{tabular}




\begin{tabular}{|c|c|c|c|c|c|c|c|c|}
\hline \multicolumn{4}{|c|}{ Trial details } & \multicolumn{2}{|c|}{ Subjects } & \multicolumn{3}{|c|}{ Between-group differences } \\
\hline Trial & Groups & Dosage & Design & No. & Details & Outcomes & $\begin{array}{l}\text { Statistical } \\
\text { significance }\end{array}$ & Clinical significance \\
\hline $\begin{array}{l}\text { Needham- } \\
\text { Shropshire } \\
\text { et al. } \\
\text { PEDro }=3 \\
\text { Blinding = no }\end{array}$ & $\begin{array}{l}\text { 1. Arm cranking+ES } \\
\text { 2. Arm cranking } \\
\text { 3. } 4 \text { weeks of arm } \\
\text { cranking+ ES followed } \\
\text { by } 4 \text { weeks of arm } \\
\text { cranking }\end{array}$ & $\begin{array}{l}20 \text { min } \\
3 \text { times per week } \\
8 \text { weeks }\end{array}$ & Between-subject & 43 & $\begin{array}{l}\text { Chronic } \\
\text { Not specified } \\
\text { Tetraplegia }\end{array}$ & $\begin{array}{l}\text { Combined upper limb ASIA } \\
\text { motor scores. }\end{array}$ & ss & Not categorized \\
\hline \multirow[t]{4}{*}{$\begin{array}{l}\text { Taylor et al. }{ }^{22} \\
\text { PEDro }=5 \\
\text { Blinding = no }\end{array}$} & $\begin{array}{l}\text { 1. Arm cranking } \\
\text { 2. No intervention }\end{array}$ & $\begin{array}{l}0.5 \mathrm{~h} \\
5 \text { times per week } \\
8 \text { weeks }\end{array}$ & Between-subject & 10 & $\begin{array}{l}\text { Chronic } \\
\text { Mixed } \\
\text { T6 and below }\end{array}$ & $\begin{array}{l}\text { Slow twitch fibre area of triceps, } \\
\text { blood lactate concentration (peak). }\end{array}$ & sS & Clearly important \\
\hline & & & & & & Heart rate (resting), weight. & SS & Inconclusive \\
\hline & & & & & & $\begin{array}{l}\text { Forced vital capacity, peak } \\
\text { oxygen consumption, triceps } \\
\text { muscle fibre distribution, fast } \\
\text { twitch fibre area of triceps, } \\
\text { blood lactate concentration } \\
\text { (resting), arm girths, skinfolds, } \\
\text { heart rate (maximal) }\end{array}$ & No SS & Inconclusive \\
\hline & & & & & & $\begin{array}{l}\text { Forced expiratory volume } \\
\text { for } 1 \mathrm{~s} \text {. }\end{array}$ & No SS & Insufficient data \\
\hline \multicolumn{9}{|l|}{ Gait training } \\
\hline $\begin{array}{l}\text { Dobkin et al. } .^{23} \\
\text { PEDro }=7\end{array}$ & $\begin{array}{l}\text { 1. Weight-supported } \\
\text { treadmill training }\end{array}$ & $\begin{array}{l}1 \mathrm{~h} \\
5 \text { times per week }\end{array}$ & Between-subject & 146 & $\begin{array}{l}\text { Acute } \\
\text { Incomplete }\end{array}$ & Walking speed. & No SS & Inconclusive \\
\hline Blinding $=$ yes & $\begin{array}{l}\text { 2. Conventional gait } \\
\text { training }\end{array}$ & 12 weeks & & & C4-L3 & $\begin{array}{l}\text { FIM-L, 6-min walk test, Berg } \\
\text { balance, WISCI, lower extremity } \\
\text { motor score, Ashworth scale, } \\
\text { quality of life. }\end{array}$ & No SS & Not categorized \\
\hline $\begin{array}{l}\text { Field-Fote et al. }{ }^{24} \\
\text { PEDro }=5 \\
\text { Blinding = no }\end{array}$ & $\begin{array}{l}\text { 1. Weight-supported } \\
\text { treadmill training+ES } \\
\text { 2. Conventional gait } \\
\text { training+ES } \\
\text { 3. Weight-supported } \\
\text { treadmill training } \\
\text { 4. Robotic walking }\end{array}$ & $\begin{array}{l}1 \mathrm{~h} \\
5 \text { times per week } \\
12 \text { weeks }\end{array}$ & Between-subject & 27 & $\begin{array}{l}\text { Chronic } \\
\text { Incomplete } \\
\text { C3-T10 }\end{array}$ & $\begin{array}{l}\text { Walking speed (short and } \\
\text { long bout), step length, step } \\
\text { length ratio. }\end{array}$ & No SS & Inconclusive \\
\hline $\begin{array}{l}\text { Harvey et al. }{ }^{25} \\
\text { PEDro }=3\end{array}$ & $\begin{array}{l}\text { 1. Gait training with } \\
\text { IRGOs }\end{array}$ & $\begin{array}{l}2-3 \mathrm{~h} \\
2-3 \text { times per week }\end{array}$ & Crossover & 10 & $\begin{array}{l}\text { Chronic } \\
\text { Motor complete }\end{array}$ & Walking speed (flat and ramps). & ss & Clearly important \\
\hline \multirow[t]{2}{*}{ Blinding $=$ no } & $\begin{array}{l}\text { 2. Gait training with } \\
\text { walkabout orthoses }\end{array}$ & 8 weeks & & & T9-T12 & $\begin{array}{l}\text { Assistance with moving from } \\
\text { sit to stand and walking } \\
\text { on ramps. }\end{array}$ & ss & Not categorized \\
\hline & & & & & & $\begin{array}{l}\text { Assistance with donning and } \\
\text { doffing, walking on the flat } \\
\text { and negotiating stairs and curbs. }\end{array}$ & No SS & Not categorized \\
\hline \multirow[t]{3}{*}{$\begin{array}{l}\text { Meenakshi et al. }{ }^{26} \\
\text { PEDro }=2 \\
\text { Blinding }=\text { no }\end{array}$} & $\begin{array}{l}\text { 1. Gait training with } \\
\text { medially linked } \\
\text { bilateral KAFOs }\end{array}$ & $\begin{array}{l}40 \text { min } \\
5 \text { times per week } \\
4 \text { weeks }\end{array}$ & Between-subject & 29 & $\begin{array}{l}>3 \text { months } \\
\text { Motor complete } \\
\text { T8-T12 }\end{array}$ & $\begin{array}{l}\text { Walking speed, degree } \\
\text { of toe out. }\end{array}$ & ss & Clearly important \\
\hline & $\begin{array}{l}\text { 2. Gait training with } \\
\text { bilateral KAFOs }\end{array}$ & & & & & Walking cadence, stride length. & SS & Inconclusive \\
\hline & & & & & & Walking Index for $\mathrm{SCl}$. & $\begin{array}{l}\text { Not } \\
\text { provided }\end{array}$ & Insufficient data \\
\hline
\end{tabular}




\begin{tabular}{|c|c|c|c|c|c|c|c|c|}
\hline \multicolumn{4}{|c|}{ Trial details } & \multicolumn{2}{|c|}{ Subjects } & \multicolumn{3}{|c|}{ Between-group differences } \\
\hline Trial & Groups & Dosage & Design & No. & Details & Outcomes & $\begin{array}{l}\text { Statistical } \\
\text { significance }\end{array}$ & Clinical significance \\
\hline \multirow[t]{2}{*}{$\begin{array}{l}\text { Postans et al. }{ }^{27} \\
\text { PEDro }=3 \\
\text { Blinding = no }\end{array}$} & $\begin{array}{l}\text { 1. Weight-supported } \\
\text { treadmill training+ES } \\
\text { 2. Conventional gait } \\
\text { training }\end{array}$ & $\begin{array}{l}1 \mathrm{~h} \\
5 \text { times per week } \\
4 \text { weeks }\end{array}$ & Crossover & 14 & $\begin{array}{l}\text { Acute } \\
\text { Motor incomplete } \\
\text { C4-T9 }\end{array}$ & $\begin{array}{l}\text { Walking endurance, walking } \\
\text { speed, walking cadence, } \\
\text { walking stride length. }\end{array}$ & Not provided & Inconclusive \\
\hline & & & & & & $\begin{array}{l}\text { Observational Gait Assessment, } \\
\text { Ashworth scale (major muscle } \\
\text { groups of lower limbs), } \\
\text { joint range of motion } \\
\text { (6 lower limb joints), strength } \\
\text { (12 lower limb muscle groups). }\end{array}$ & Not provided & Insufficient data \\
\hline \multirow[t]{2}{*}{$\begin{array}{l}\text { Hand therapy } \\
\text { Beekhuizen et al. }{ }^{28} \\
\text { PEDro }=5 \\
\text { Blinding = no }\end{array}$} & $\begin{array}{l}\text { 1. Massed practice } \\
\text { of hand } \\
\text { activities+somatosen- } \\
\text { sory stimulation }\end{array}$ & $\begin{array}{l}2 \mathrm{~h} \\
5 \text { times per week } \\
3 \text { weeks }\end{array}$ & Between-subject & 10 & $\begin{array}{l}\text { Chronic } \\
\text { Incomplete } \\
\text { C5-C7 }\end{array}$ & $\begin{array}{l}\text { Wolf Motor Function Test, } \\
\text { pinch grip, Jebsen Hand } \\
\text { Function Test. }\end{array}$ & ss & Not categorized \\
\hline & $\begin{array}{l}\text { 2. Massed practice } \\
\text { of hand activities }\end{array}$ & & & & & $\begin{array}{l}\text { Motor-evoked potential } \\
\text { thresholds, motor-evoked } \\
\text { potential amplitudes. }\end{array}$ & No SS & Not categorized \\
\hline $\begin{array}{l}\text { Kohlmeyer et al. } \\
\text { PEDro =4 } \\
\text { Blinding =yes }\end{array}$ & $\begin{array}{l}\text { 1. Wrist extensor } \\
\text { exercises+biofeed- } \\
\text { back+ES } \\
\text { 2. Conventional hand } \\
\text { therapy } \\
\text { 3. Wrist extensor } \\
\text { exercises+biofeedback } \\
\text { 4. Wrist extensor } \\
\text { exercises+ES }\end{array}$ & $\begin{array}{l}20 \text { min } \\
5 \text { times per week } \\
5-6 \text { weeks }\end{array}$ & Between-subject & 60 & $\begin{array}{l}\text { Acute } \\
\text { Mixed } \\
\text { C4-C6 }\end{array}$ & $\begin{array}{l}\text { Manual muscle test, ability } \\
\text { to self-feed ( } 4 \text { tests). }\end{array}$ & No SS & Insufficient data \\
\hline $\begin{array}{l}\text { Popovic et al. } \\
\text { PEDro }=3 \\
\text { Blinding }=\text { no }\end{array}$ & $\begin{array}{l}\text { 1. Conventional hand } \\
\text { therapy+ES } \\
\text { 2. Conventional hand } \\
\text { therapy }\end{array}$ & $\begin{array}{l}45 \text { min } \\
5 \text { times per week } \\
12 \text { weeks }\end{array}$ & Between-subject & 21 & $\begin{array}{l}\text { Acute } \\
\text { Mixed } \\
\text { C3-C7 }\end{array}$ & $\begin{array}{l}\text { Spinal Cord Independence } \\
\text { Measure, FIM, hand function } \\
\text { (total and } 5 \text { subtotals each for } \\
\text { completes and incompletes). }\end{array}$ & No SS & Not categorized \\
\hline \multicolumn{9}{|c|}{ Stretch-based interventions } \\
\hline $\begin{array}{l}\text { Ben et } a l^{31} \\
\text { PEDro }=8\end{array}$ & $\begin{array}{l}\text { 1. Weight-bearing } \\
\text { standing }\end{array}$ & $\begin{array}{l}0.5 \mathrm{~h} \\
3 \text { times per week }\end{array}$ & Within-subject & 20 & $\begin{array}{l}\text { Acute } \\
\text { Mixed }\end{array}$ & Passive ankle range of motion. & SS & Inconclusive \\
\hline Blinding $=$ yes & $\begin{array}{l}\text { 2. Non weight-bearing } \\
\text { standing }\end{array}$ & 12 weeks & & & $\begin{array}{l}\text { Wheelchair- } \\
\text { dependent }\end{array}$ & $\begin{array}{l}\text { Total proximal femur bone } \\
\text { mineral density. }\end{array}$ & No ss & Inconclusive \\
\hline $\begin{array}{l}\text { Crowe et al. }{ }^{10} \\
\text { PEDro }=5\end{array}$ & $\begin{array}{l}\text { 1. Conventional } \\
\text { care+upper limb }\end{array}$ & $\begin{array}{l}0.75 \mathrm{~h} \\
5 \text { times per week }\end{array}$ & Between-subject & 39 & $\begin{array}{l}\text { Acute } \\
\text { Complete }\end{array}$ & Pain. & No ss & Inconclusive \\
\hline Blinding $=$ yes & $\begin{array}{l}\text { positioning } \\
\text { 2. Conventional care }\end{array}$ & Mean 6 weeks & & & $\mathrm{C} 2-\mathrm{C} 7$ & $\begin{array}{l}\text { Passive shoulder range of motions } \\
\text { (8 tests), FIM, time in wheelchair. }\end{array}$ & No ss & Insufficient data \\
\hline \multirow[t]{2}{*}{$\begin{array}{l}\text { Harvey et al. } \\
\text { PEDro }=8 \\
\text { Blinding = yes }\end{array}$} & $\begin{array}{l}\text { 1. Ankle stretch } \\
\text { 2. No intervention }\end{array}$ & $\begin{array}{l}0.5 \mathrm{~h} \\
5-7 \text { times per week } \\
4 \text { weeks }\end{array}$ & Within-subject & 14 & $\begin{array}{l}\text { Acute } \\
\text { Not specified } \\
\text { Wheelchair- } \\
\text { dependent }\end{array}$ & $\begin{array}{l}\text { Ankle range of motion with } \\
\text { knee extended and flexed } \\
\text { ( } 3 \text { tests). }\end{array}$ & No ss & Ineffective \\
\hline & & & & & & Ankle stiffness ( 3 tests). & No SS & Inconclusive \\
\hline
\end{tabular}




\begin{tabular}{|c|c|c|c|c|c|c|c|c|}
\hline \multicolumn{4}{|c|}{ Trial details } & \multicolumn{2}{|c|}{ Subjects } & \multicolumn{3}{|c|}{ Between-group differences } \\
\hline Trial & Groups & Dosage & Design & No. & Details & Outcomes & $\begin{array}{l}\text { Statistical } \\
\text { significance }\end{array}$ & Clinical significance \\
\hline $\begin{array}{l}\text { Harvey et } \text { al. }^{33} \\
\text { PEDro }=8 \\
\text { Blinding =yes }\end{array}$ & $\begin{array}{l}\text { 1. Hamstring stretch } \\
\text { 2. No intervention }\end{array}$ & $\begin{array}{l}0.5 \mathrm{~h} \\
5 \text { times per week } \\
4 \text { weeks }\end{array}$ & Within-subject & 16 & $\begin{array}{l}\text { Acute } \\
\text { Not specified } \\
\text { Lower limb } \\
\text { paralysis }\end{array}$ & Passive hip range of motion. & No SS & Ineffective \\
\hline \multicolumn{9}{|l|}{ Hand splinting } \\
\hline $\begin{array}{l}\text { DiPasquale-Lehnerz } \\
\text { et } \text { al. }^{34} \\
\text { PEDro }=3 \\
\text { Blinding }=\text { no }\end{array}$ & $\begin{array}{l}\text { 1. Hand splinting } \\
\text { 2. No intervention }\end{array}$ & $\begin{array}{l}8 \mathrm{~h} \\
7 \text { times per week } \\
3 \text { months }\end{array}$ & Between-subject & 13 & $\begin{array}{l}\text { Acute } \\
\text { Complete } \\
\text { C6 }\end{array}$ & $\begin{array}{l}\text { Jebsen Hand Function Test, } \\
\text { grip strength, wrist and hand } \\
\text { range of motion ( } 5 \text { tests), } \\
\text { wrist strength. }\end{array}$ & No SS & Insufficient data \\
\hline $\begin{array}{l}\text { Harvey et } \text { al. }^{35} \\
\text { PEDro }=8 \\
\text { Blinding }=\text { yes }\end{array}$ & $\begin{array}{l}\text { 1. Thumb splinting } \\
\text { 2. No intervention }\end{array}$ & $\begin{array}{l}8 \mathrm{~h} \\
7 \text { times per week } \\
12 \text { weeks }\end{array}$ & Within-subject & 20 & $\begin{array}{l}\text { Chronic } \\
\text { Not specified } \\
\text { C4-C7 }\end{array}$ & $\begin{array}{l}\text { Carpometacarpal range of } \\
\text { motion ( } 3 \text { tests). }\end{array}$ & No Ss & Ineffective \\
\hline \multirow{4}{*}{$\begin{array}{l}\text { Acupuncture } \\
\text { Dyson-Hudson } \\
\text { et al. }{ }^{36} \\
\text { PEDro }=5 \\
\text { Blinding =yes }\end{array}$} & & & & & & & & \\
\hline & $\begin{array}{l}\text { 1. Acupuncture } \\
\text { 2. Trager treatment }\end{array}$ & $\begin{array}{l}10 \text { treatments } \\
\text { Over } 5 \text { weeks }\end{array}$ & Between-subject & 24 & $\begin{array}{l}\text { Chronic } \\
\text { Mixed } \\
\text { C6-T12 }\end{array}$ & $\begin{array}{l}\text { WUSPI, pain (10-point scale), } \\
\text { shoulder ranges of motion } \\
\text { (4 tests). }\end{array}$ & No SS & Inconclusive \\
\hline & & & & & & Pain (6-point scale). & No SS & Not categorized \\
\hline & & & & & & Analgesic intake (3 tests). & $\begin{array}{l}\text { Not } \\
\text { provided }\end{array}$ & Inconclusive \\
\hline $\begin{array}{l}\text { Dyson-Hudson } \\
\text { et al. } .^{37} \\
\text { PEDro }=8 \\
\text { Blinding =yes }\end{array}$ & $\begin{array}{l}\text { 1. Acupuncture } \\
\text { 2. Sham acupuncture }\end{array}$ & $\begin{array}{l}10 \text { treatments } \\
\text { Over } 5 \text { weeks }\end{array}$ & Between-subject & 23 & $\begin{array}{l}\text { Chronic } \\
\text { Not specified } \\
\text { Not specified }\end{array}$ & WUSPI, pain. & No SS & Inconclusive \\
\hline \multirow[t]{3}{*}{$\begin{array}{l}\text { Wong et al. } .^{38} \\
\text { PEDro }=4 \\
\text { Blinding }=\text { no }\end{array}$} & $\begin{array}{l}\text { 1. Standard } \\
\text { care+acupuncture } \\
\text { 2. Standard care }\end{array}$ & $\begin{array}{l}30 \text { min } \\
3 \text { times per week } \\
8 \text { weeks }\end{array}$ & Between-subject & 100 & $\begin{array}{l}\text { Acute } \\
\text { Motor complete } \\
\text { Not specified }\end{array}$ & $\begin{array}{l}\text { ASIA motor, ASIA pinprick, } \\
\text { ASIA light touch. }\end{array}$ & ss & Clearly important \\
\hline & & & & & & FIM total. & SS & Inconclusive \\
\hline & & & & & & ASIA impairment scale. & sS & Not categorized \\
\hline \multicolumn{9}{|l|}{ Other therapies } \\
\hline $\begin{array}{l}\text { Curtis et al. }{ }^{39} \\
\text { PEDro }=3 \\
\text { Blinding = no }\end{array}$ & $\begin{array}{l}\text { 1. Shoulder exercises } \\
\text { 2. No intervention }\end{array}$ & $\begin{array}{l}0.5 \mathrm{~h} \\
7 \text { times per week } \\
6 \text { months }\end{array}$ & Between-subject & 42 & $\begin{array}{l}\text { Chronic } \\
\text { Not specified } \\
\text { C6 and below } \\
(n=35+7 \text { non-SCI } \\
\text { conditions })\end{array}$ & WUSPI. & No SS & Inconclusive \\
\hline \multirow[t]{3}{*}{$\begin{array}{l}\text { Diego et al. }{ }^{9} \\
\text { PEDro }=4 \\
\text { Blinding }=\text { no }\end{array}$} & $\begin{array}{l}\text { 1. Massage for upper } \\
\text { limbs and trunk } \\
\text { 2. Head and upper } \\
\text { limb range of motion }\end{array}$ & $\begin{array}{l}40 \text { min } \\
2 \text { times per week } \\
5 \text { weeks }\end{array}$ & Between-subject & 20 & $\begin{array}{l}\text { Chronic } \\
\text { Not specified } \\
\text { C5-C7 }\end{array}$ & $\begin{array}{l}\text { Depression, manual muscle } \\
\text { test ( } 14 \text { muscles), wrist flexion } \\
\text { and extension range of motion. }\end{array}$ & SS & Inconclusive \\
\hline & exercises & & & & & $\begin{array}{l}\text { Barthel Index, upper limb } \\
\text { range of motion ( } 4 \text { tests). }\end{array}$ & No SS & Inconclusive \\
\hline & & & & & & $\begin{array}{l}\text { Elbow flexion range } \\
\text { of motion. }\end{array}$ & No SS & Ineffective \\
\hline
\end{tabular}




\begin{tabular}{|c|c|c|c|c|c|c|c|c|}
\hline \multicolumn{4}{|c|}{ Trial details } & \multicolumn{2}{|c|}{ Subjects } & \multicolumn{3}{|c|}{ Between-group differences } \\
\hline Trial & Groups & Dosage & Design & No. & Details & Outcomes & $\begin{array}{l}\text { Statistical } \\
\text { significance }\end{array}$ & Clinical significance \\
\hline $\begin{array}{l}\text { Klose et al. }{ }^{40} \\
\text { PEDro }=4 \\
\text { Blinding }=\text { yes }\end{array}$ & $\begin{array}{l}\text { 1. Elbow and wrist } \\
\text { exercises+ES } \\
\text { 2. Conventional therapy } \\
\text { 3. Elbow and wrist } \\
\text { exercises+biofeedback }\end{array}$ & $\begin{array}{l}\text { Not specified } \\
3 \text { times per week } \\
16 \text { weeks }\end{array}$ & Between-subject & 43 & $\begin{array}{l}\text { Chronic } \\
\text { Incomplete } \\
\text { C4-C6 }\end{array}$ & $\begin{array}{l}\text { Summed self-care functional } \\
\text { score, motor score, summed } \\
\text { mobility score, EMG activity. }\end{array}$ & No SS & Insufficient data \\
\hline $\begin{array}{l}\text { Klose et al. }{ }^{41} \\
\text { PEDro }=5 \\
\text { Blinding }=\text { no }\end{array}$ & $\begin{array}{l}\text { 1. Conventional } \\
\text { therapy+ES+ } \\
\text { biofeedback } \\
\text { for upper limbs } \\
\text { 2. Conventional } \\
\text { therapy+ES for upper } \\
\text { limbs }\end{array}$ & $\begin{array}{l}1.75 \mathrm{~h} \\
3 \text { times per week } \\
12 \text { weeks }\end{array}$ & Between-subject & 31 & $\begin{array}{l}\text { Chronic } \\
\text { Not specified } \\
\text { C5-C7 }\end{array}$ & $\begin{array}{l}\text { Modified ASIA motor score, } \\
\text { summed score on battery } \\
\text { of functional tests. }\end{array}$ & No SS & Inconclusive \\
\hline $\begin{array}{l}\text { Lechner et al. } \\
\text { PEDro }=4 \\
\text { Blinding =yes }\end{array}$ & $\begin{array}{l}\text { 1. Hippotherapy } \\
\text { (horse therapy) } \\
\text { 2. Exercises while sitting } \\
\text { on a Bobath roll } \\
\text { 3. Exercises while sitting } \\
\text { on a rocking seat }\end{array}$ & $\begin{array}{l}25 \text { min } \\
2 \text { times per week } \\
4 \text { weeks }\end{array}$ & Crossover & 12 & $\begin{array}{l}\text { Chronic } \\
\text { Motor complete } \\
\text { C7-T10 }\end{array}$ & $\begin{array}{l}\text { Ashworth scale, spasticity } \\
\text { self-report, self-rated } \\
\text { well-being. }\end{array}$ & No SS & Insufficient data \\
\hline $\begin{array}{l}\text { Lopes et al. }{ }^{42} \\
\text { PEDro }=2 \\
\text { Blinding }=\text { no }\end{array}$ & $\begin{array}{l}\text { 1. Graded tilting+upper } \\
\text { limb exercises } \\
\text { 2. Graded tilting }\end{array}$ & $\begin{array}{l}10 \text { treatments } \\
\text { Over } 10 \text { days }\end{array}$ & Between-subject & 12 & $\begin{array}{l}\text { Acute } \\
\text { Not specified } \\
\text { C5 and below }\end{array}$ & $\begin{array}{l}\text { Tolerated angle of tilt, } \\
\text { heart rate, blood pressure. }\end{array}$ & No SS & Insufficient data \\
\hline $\begin{array}{l}\text { Warden et al. }{ }^{43} \\
\text { PEDro }=6\end{array}$ & $\begin{array}{l}\text { 1. Real ultrasound to } \\
\text { the heel }\end{array}$ & $\begin{array}{l}20 \text { min } \\
5 \text { times per week }\end{array}$ & Within-subject & 15 & $\begin{array}{l}\text { Acute } \\
\text { Motor complete }\end{array}$ & Bone mineral content. & No ss & Ineffective \\
\hline Blinding = yes & $\begin{array}{l}\text { 2. Sham ultrasound } \\
\text { to the heel }\end{array}$ & 6 weeks & & & Above $\mathrm{T} 12$ & $\begin{array}{l}\text { Bone ultrasound } \\
\text { attenuation, speed } \\
\text { of sound. }\end{array}$ & No ss & Inconclusive \\
\hline
\end{tabular}

Abbreviations: ASIA, American Spinal Injury Association; ES, electrical simulation; FIM, Functional Independence Measure; FIM-L, Functional Independence Measure locomotor walking score; IRGOs, Isocentric Reciprocal Gait Orthoses; KAFO, knee-ankle-foot orthoses; WUSPI, Wheelchair User's Shoulder Pain Index.

The number of subjects (no.) refers to the number of subjects who started the trial. Subject details include time since injury, completeness of injury and level of injury (if provided). 'Acute' indicates injuries less than 1 year and 'chronic' indicates injuries more than 1 year. 'Mixed' indicates complete and incomplete injuries. The between-group statistical significance (statistical significance), as stated by the authors of the trials, are indicated for each outcome where SS indicates statistical significance and no SS indicates no statistical significance. The clinical significance refers to the $95 \% \mathrm{Cl}$ of the between-group difference with respect to minimally important between-group differences. The clinical significance refers to the $95 \% \mathrm{Cl}$ of the between-group difference with respect to minimally important between-group differences. Between-group differences reflecting clearly important treatment effects (indicated by the term clearly important) or ineffective treatments (indicated by the term ineffective) are bolded to highlight the conclusively positive and negative findings, respectively (see Methods section and Figure 1). 
Fourteen trials included people with recent SCI (that is, less than 1 year), 16 trials included people with chronic SCI (that is, more than 1 year) and one trial did not specify time since injury other than to say subjects were more than 3 months since injury. ${ }^{26}$ There were approximately equal numbers of trials including people with complete and incomplete SCI, and people with tetraplegia and paraplegia. The most common outcome measures were walking speed (five trials), joint range of motion (eight trials) and exercise capacity (four trials).

The 31 trials were broadly grouped into seven categories after taking into account the key purpose of the trial and the nature of the intervention (see Table 1). Meta-analyses were not performed because of the clinical heterogeneity between trials.

The key findings summarized in Table 1 are:

(1) Fitness and strength training: Seven trials investigated the effectiveness of arm or leg exercise. Six trials involved active exercise of the upper limbs with or without electrical simulation (ES), ${ }^{7,18-22}$ and one trial involved ES-driven exercise for the paralyzed lower limbs. ${ }^{17}$ Six of the seven trials reported a statistically significant between-group difference on at least one outcome measure. ${ }^{17-22}$ However, only three trials had clearly important between-group differences. The key findings from these three trials were that ES-driven exercise (versus no intervention) of the paralyzed lower limbs increased lower limb lean body mass; ${ }^{17}$ strength and fitness training (versus education and relaxation) decreased pain; ${ }^{19}$ and arm-cranking exercise (versus no intervention) increased the proportion of slow twitch muscle fibres in the triceps muscles. ${ }^{22}$ The size of the between-group differences could not be ascertained in the other three trials with statistically significant between-group differences. ${ }^{18,20,21}$

(2) Gait training: Five trials assessed the effectiveness of gait training either with weight-supported systems ${ }^{23,24,27}$ or with orthoses. ${ }^{25,26}$ Two trials included ES. ${ }^{24,27}$ Statistically significant and clearly important between-group differences were reported in two trials on at least one outcome measure. One of these trials found that the subjects walked faster with medially linked knee-anklefoot orthoses (KAFO) than unlinked knee-ankle-foot orthoses, ${ }^{26}$ and the other trial found that the subjects walked faster with isocentric reciprocal gait orthoses than medially linked knee-ankle-foot orthoses. ${ }^{25}$ The other three trials ${ }^{23,24,27}$ compared the relative effectiveness of two or more of the following interventions: weight-supported treadmill training with or without electrical stimulation, conventional gait training with or without electrical stimulation and robotic walking. All three were inconclusive despite, in one trial, the

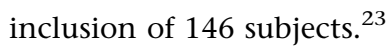

(3) Hand therapy: Three trials compared biofeedback, ${ }^{29} \mathrm{ES}^{30}$ or somatosensory stimulation ${ }^{28}$ of the hand with conventional hand therapy. One $\operatorname{trial}^{28}$ reported a statistically significant between-group difference on functional measures of hand function and pinch grip but the data were skewed, so the size of the betweengroup difference could not be quantified. This trial examined the added benefit of somatosensory stimulation with massed practice of hand activities in people with incomplete tetraplegia. The other two trials did not report statistically significant between-group differences. ${ }^{29,30}$

(4) Stretch-based interventions: Four trials examined the effectiveness of different stretch-based interventions on range of motion and shoulder pain. ${ }^{10,31-33}$ Two trials demonstrated that stretch-based interventions for range of motion were ineffective (versus no intervention or conventional care $)^{32,33}$ and two trials were inconclusive. ${ }^{10,31}$

(5) Hand splinting: Two trials examined the effect of hand splints in people with tetraplegia. ${ }^{34,35}$ One trial demonstrated that splinting the thumb was ineffective for decreasing the extensibility of the flexor pollicis longus muscle (versus no splint), ${ }^{35}$ and the other trial did not provide sufficient data to determine between-group differences. ${ }^{34}$

(6) Acupuncture: Three trials examined the effect of acupuncture. $^{36-38}$ One trial, notable for its large size, demonstrated that the addition of acupuncture to standard care administered soon after injury improved strength and sensation. ${ }^{38}$ The other two trials investigated the effectiveness of acupuncture for shoulder pain and were inconclusive.

(7) Other therapies: Seven trials examined a range of different therapies including the effect of shoulder exercises or massage for mobility and depression, ${ }^{9}$ ES or biofeedback for function, ${ }^{40,41}$ hippotherapy for spasticity, ${ }^{8}$ upper limb exercise with graded tilting for postural hypotension, ${ }^{42}$ ultrasound for bone loss ${ }^{43}$ and stretches and strengthening exercises for shoulder pain. ${ }^{39}$ A trial investigating massage (versus head and upper limb range of motion exercises) had mixed results reporting between-group statistical differences for some outcomes but treatment ineffectiveness for the others. ${ }^{9}$ Ultrasound (versus sham ultrasound) was ineffective for preventing bone loss. ${ }^{43}$ There were insufficient data or inconclusive evidence to support ES or biofeedback for function, ${ }^{40,41}$ hippotherapy for spasticity ${ }^{8}$ or stretches and strengthening exercises for shoulder pain. ${ }^{39}$

\section{Discussion}

It is somewhat surprising that only 31 randomized controlled trials have investigated the effectiveness of different physical interventions for people with SCI. Of the 31 identified trials, six reported between-group and clearly important differences on at least one outcome measure. ${ }^{17,19,22,25,26,38}$ Six more trials reported between-group statistical differences but either the results were inconclusive $e^{9,31}$ or the size of the between-group differences could not be determined. ${ }^{18,21,20,28}$ The majority of trials either did not report or did not find between-group statistical differences and were inconclusive $7,8,10,23,24,27,29,30,34,36,37,39-42$ or demonstrated treatment ineffectiveness. ${ }^{32,33,35,43}$

The interpretation of this systematic review is partly dependent on the definition of minimally important between-group differences. ${ }^{16}$ Ideally, researchers articulate minimally important differences for each outcome before 
the commencement of the trials. However, this was rarely done. The failure to do this not only potentially introduces bias to the interpretation of results but also leads to a reliance on statistical significance without taking into account the size of treatment effects. We needed a minimally important difference for each study to provide a meaningful interpretation of results and to summarize the large number of outcomes (it was not feasible to provide the betweengroup differences and 95\% CIs of the 200 outcomes reported in this review). We considered using a distribution-based approach where the size of the treatment effect is normalized in relation to sample variance (for example, Cohen's d). However, these approaches are strongly influenced by the heterogeneity of the samples. ${ }^{44}$ In addition, they do not give a clear indication of the importance of treatment effects. ${ }^{45}$ For these reasons, we opted a priori to nominate a meaningful between-group difference equivalent to $10 \%$ of subjects' initial status unless otherwise articulated by the investigators. The value of $10 \%$ was somewhat arbitrary but probably overestimates rather than underestimates treatment effectiveness. It is unlikely that the time, cost and inconvenience associated with most interventions administered over prolonged periods of time can be justified unless the added benefit is equivalent to at least $10 \%$ of initial status.

The results of this systematic review provide some support for different strength and fitness training programmes with and without electrical stimulation. ${ }^{17,19,22}$ The results also indicate the relative effectiveness of gait training with orthoses. $^{25,26}$ The largest trial demonstrated that acupuncture improved strength and sensation. ${ }^{38}$ However, the findings of all these trials need to be interpreted with caution. None blinded assessors, concealed allocation or had PEDro scores greater than 5 (our PEDro ratings are lower than those given by other reviewers ${ }^{3}$ but consistent with the independent ratings of the Centre for Evidence-based Physiotherapy; ${ }^{5}$ the original authors of the PEDro scale). In addition, some trials had numerous outcome measures and statistical comparisons without adjustments for the increased likelihood of type I statistical errors (that is, finding statistical between-group differences by chance). The number of statistical comparisons per trial can be gauged from Table 1. This table probably understates this potential problem because some analyses may not have been reported and some investigators reported additional results in duplicate publications not included in this review. Interestingly, very few trials reported clearly important treatment effects on activity limitations and participation restrictions. Instead, the treatment effects were predominantly measured at the impairment level. This may be because impairments are often more directly responsive to interventions and less influenced by the array of variables affecting activity limitations and participation restrictions. ${ }^{46,47}$

Four trials indicated that treatments were ineffective $^{32,33,35,43}$ These trials looked at the effectiveness of stretch for the management of contractures, ${ }^{32,33}$ splinting for the promotion of muscle shortening ${ }^{35}$ and ultrasound for the treatment of bone loss. ${ }^{43}$ The negative results of these trials could reflect design weaknesses such as poor inclusion criteria and inappropriate treatment dosage. However, these trials had a median (interquartile range) PEDro score of 8 (7.5-8). The results of these trials should therefore at least raise questions about the effectiveness of interventions, some of which are still routinely provided to people with SCI.

The majority of trials in this systematic review compared two or more types of interventions, which often differed only in subtle ways. In the absence of between-group differences, some investigators performed statistical analyses on pre- to post-intervention data. Significant change over time in groups was then attributed to the effectiveness of all interventions. This approach is flawed. Change over time can be due to any number of factors and does not provide good evidence for treatment effectiveness (for example, change can be due to natural recovery or exposure to the testing protocol). ${ }^{48}$ Proper estimates of treatment effectiveness can only come from between-group differences.

The trials identified in this systematic review included an array of people with different attributes and types of SCI. Some trials had very homogeneous subjects whereas others did not. There are clear advantages associated with restricting inclusion criteria to very specific types of subjects, provided it is known which types of subjects are most responsive to the intervention. Subjects with similar attributes are more likely to respond to the intervention in a consistent way, thereby reducing variability and increasing the likelihood of detecting between-group differences (that is, increasing statistical power). However, it is not always known which groups of subjects are most likely to respond to interventions. In this situation, it is not unreasonable to adopt a pragmatic approach and follow clinical practice, in which case patients in whom the treatments are typically administered in the clinical setting are used as subjects for trials. ${ }^{48}$

Most trials identified by this review were inconclusive. That is, the 95\% CIs associated with the between-group differences spanned the minimally important differences (see Figure 1). The majority of these results were also statistically insignificant (see Table 1). Statistically insignificant findings do not provide good evidence for treatment ineffectiveness unless the upper end of the 95\% CI falls short of the minimally worthwhile treatment effect. It was therefore not clear from the majority of trials whether the treatments were or were not effective. ${ }^{15}$

Inconclusive results are a common problem in trials investigating physical interventions. There are a number of explanations for this but it is primarily due to the difficulties associated with demonstrating modest treatment effects in heterogeneous subjects. ${ }^{13}$ It is also due to the large number of factors influencing outcomes such as completeness of injury, time since injury and level of injury. These factors do not systematically bias results, provided subjects are randomized, but they do generate noise making it difficult to isolate the effects of interventions. The obvious way to reduce the likelihood of inconclusive results is to increase sample size. However, it is difficult to recruit large numbers of homogeneous subjects without extensive financial support and multi-centred co-operation. Trials can also reduce the likelihood of inconclusive results by limiting the number of experimental groups. This strategy increases the number of subjects in each arm of the trial. Trials that measure at the 
impairment level also decrease the likelihood of inconclusive results. Needless to say that the relevance of treatment effects on impairments is increased when accompanied by evidence about treatment effects on activity limitations and participation restrictions.

Interestingly, some of the higher quality trials had more problems with inconclusive results than some of the lower quality trials. Perhaps this is partly due to the inherent biases of low-quality trials. Low-quality trials tend to overstate treatment effectiveness. ${ }^{49-52}$ High-quality trials minimize bias and therefore provide a more robust and honest reflection of the uncertainty around estimates of treatment effectiveness. Inconclusive results are undesirable; however, they are still valuable provided they come from high-quality trials. Results of this kind can be pooled in rigorous metaanalyses. ${ }^{15}$ Future meta-analyses may provide our best hope for quantifying the effectiveness of some physical interventions, given the challenges of conducting adequately powered trials in this area.

The results of this systematic review provide initial evidence of the effectiveness of fitness training, strength training, gait training and acupuncture for people with SCI. However, there is still a long way to go to provide an evidence base for the wide range of physical interventions that have become standard practice. As we move forward to explore the effectiveness of new and emerging therapies, it is important that emphasis continues to be placed on highquality trials. Ideally, these trials will be adequately powered to provide conclusive findings. However, this is not always going to be possible, in which case our best hope for quantifying the effectiveness of some physical interventions may come from future meta-analyses.

\section{References}

1 Sackett DL, Strauss EE, Richardson WS, Rosenberg W, Haynes RB. Evidence-Based Medicine. How to Practice and Teach EBM, 2nd edn. Churchill Livingstone: Edinburgh, 2000.

2 Schulz K, Grimes D. The Lancet Handbook of Essential Concepts in Clinical Research 2006. Elsevier: Edinburgh.

3 Eng JJ, Teasell RW, Miller WC, Wolfe DL, Townson AF, Aubut J et al. Spinal Cord Injury Rehabilitation Evidence 2007. Vancouver.

4 Consortium for Spinal Cord Medicine. Clinical Practice Guidelines. Paralyzed Veterans of America: Washington, DC, 1999.

5 Moseley AM, Herbert RD, Sherrington C, Maher CG. Evidence for physiotherapy practice: a survey of the physiotherapy evidence based database (PEDro). Aust J Physiother 2002; 48: 43-50.

6 Dickersin K, Scherer R, Lefebvre C. Identifying relevant studies for systematic reviews. BMJ 1994; 309: 1286-1291.

7 Hartkopp A, Harridge SD, Mizuno M, Ratkevicius A, Quistorff B, Kjaer $\mathrm{M}$ et al. Effect of training on contractile and metabolic properties of wrist extensors in spinal cord-injured individuals. Muscle Nerve 2003; 27: 72-80.

8 Lechner HE, Kakebeeke TH, Hegemann D, Baumberger M. The effect of Hippotherapy on spasticity and on mental well-being of persons with spinal cord injury. Arch Phys Med Rehabil 2007; 88: 1241-1248.

9 Diego MA, Field T, Hernandez-Reif M, Hart S, Brucker B, Field T et al. Spinal cord patients benefit from massage therapy. Int $J$ Neurosci 2002; 112: 133-142.

10 Crowe J, MacKay-Lyons M, Morris H. A multi-centre, randomized controlled trial of the effectiveness of positioning on quadriplegic shoulder pain. Physiother Can 2000; 52: 266-273.
11 Deeks JJ, Higgins JPT, Altman DF (eds). Analysing and presenting results. In: Higgins JPT, Green S (eds). Cochrane Handbook for Systematic Reviews of Interventions 4.2.6 (updated September 2006); Section 8. In: The Cochrane Library, Issue 4, 2006. John Wiley and Sons, Ltd: Chichester, UJ.

12 Fleiss J. The statistical basis of meta-analysis. Stat Methods Med Res 1993; 2 : 121-145.

13 Herbert R. How to estimate treatment effect from reports of clinical trials. 1: continuous outcomes. Aust J Physiother 2000; 46: 229-235.

14 Green S, Deeks J, Savio F. Meta-analysis of continuous data: does the end justify the mean? In: 9th International Cochrane Colloquium: Lyon, France, 2001.

15 Sackett DL, Cook DJ. Can we learn anything from small trials? Ann N Y Acad Sci 1993; 703: 25-32.

16 Barrett B, Brown D, Mundt M, Brown R. Sufficiently important difference: expanding the framework of clinical significance. Med Decis Making 2005; 25: 250-261.

17 Baldi JC, Jackson RD, Moraille R, Mysiw WJ. Muscle atrophy is prevented in patients with acute spinal cord injury using functional electrical stimulation. Spinal Cord 1998; 36: 463-469.

18 de Groot PC, Hjeltnes N, Heijboer AC, Stal W, Birkeland K. Effect of training intensity on physical capacity, lipid profile and insulin sensitivity in early rehabilitation of spinal cord injured individuals. Spinal Cord 2003; 41: 673-679.

19 Hicks AL, Martin KA, Ditor DS, Latimer AE, Craven C, Bugaresti J et al. Long-term exercise training in persons with spinal cord injury: effects on strength, arm ergometry performance and psychological well-being. Spinal Cord 2003; 41: 34-43.

20 McLean KP, Skinner JS. Effect of body training position on outcomes of an aerobic training study on individuals with quadriplegia. Arch Phys Med Rehabil 1995; 76: 139-150.

21 Needham-Shropshire BM, Broton JG, Cameron TL, Klose KJ. Improved motor function in tetraplegics following neuromuscular stimulation-assisted arm ergometry. J Spinal Cord Med 1997; 20: 49-55.

22 Taylor AW, McDonell E, Brassard L. The effects of an arm ergometer training programme on wheelchair subjects. Paraplegia 1986; 24: 105-114.

23 Dobkin B, Apple D, Barbeau H, Basso M, Behrman A, Deforge D et al. Weight-supported treadmill vs over-ground training for walking after acute incomplete SCI. Neurology 2006; 66: 484-493.

24 Field-Fote EC, Lindley SD, Sherman AL. Locomotor training approaches for individuals with spinal cord injury: a preliminary report of walking-related outcomes. J Neurol Phys Ther 2005; 29: 127-137.

25 Harvey LA, Smith MB, Davis GM, Engel S. Functional outcomes attained by T9-12 paraplegic patients with the walkabout and the isocentric reciprocal gait orthoses. Arch Phys Med Rehabil 1997; 78: 706-711.

26 Meenakshi S, Chitra K. Effect of medially linked and unlinked knee immobilizer on paraplegic gait performance. Indian J Physiother Occup Ther 2007; 1: 23-31.

27 Postans NJ, Hasler JP, Granat MH, Maxwell DJ. Functional electrical stimulation to augment partial weight-bearing supported treadmill training for patients with acute incomplete spinal cord injury: a pilot study. Arch Phys Med Rehabil 2004; 85: 604-610.

28 Beekhuizen KS, Field-Fote EC. Massed practice versus massed practice with stimulation: effects on upper extremity function and cortical plasticity in individuals with incomplete cervical spinal cord injury. Neurorehabil Neural Repair 2005; 19: 33-47.

29 Kohlmeyer KM, Hill JP, Yarkony GM, Jaeger RJ. Electrical stimulation and biofeedback effect on recovery of tenodesis grasp: a controlled study. Arch Phys Med Rehabil 1996; 77: 701-706.

30 Popovic MR, Thrasher TA, Adams ME, Takes V, Zivanovic V, Tonack MI. Functional electrical therapy: retraining grasping in spinal cord injury. Spinal Cord 2006; 44: 143-151.

31 Ben M, Harvey L, Denis S, Glinsky J, Goehl G, Chee S et al. Does 12 weeks of regular standing prevent loss of ankle mobility and bone mineral density in people with recent spinal cord injuries? Aust J Physiother 2005; 51: 251-256.

32 Harvey LA, Batty J, Crosbie J, Poulter S, Herbert RD. A randomized trial assessing the effects of 4 weeks of daily 
stretching on ankle mobility in patients with spinal cord injuries. Arch Phys Med Rehabil 2000; 81: 1340-1347.

33 Harvey LA, Byak AJ, Ostrovskaya M, Glinsky J, Katte L, Herbert $\mathrm{RD}$. Randomised trial of the effects of four weeks of daily stretch on extensibility of hamstring muscles in people with spinal cord injuries. Aust J Physiother 2003; 49: 176-181.

34 DiPasquale-Lehnerz P. Orthotic intervention for development of hand function with C-6 quadriplegia. Am J Occup Ther 1994; 48: 138-144.

35 Harvey L, Simpson D, Pironello D, Glinsky J, Baillie R, Ritchie B. Does three months of nightly splinting reduce the extensibility of the flexor pollicis longus muscle in people with tetraplegia? Physiother Res Int 2007; 12: 5-13.

36 Dyson-Hudson TA, Shiflett SC, Kirshblum SC, Bowen JE, Druin EL. Acupuncture and Trager psychophysical integration in the treatment of wheelchair user's shoulder pain in individuals with spinal cord injury. Arch Phys Med Rehabil 2001; 82: 1038-1046.

37 Dyson-Hudson T, Kadar P, la Fountaine M, Emmons R, Kirshblum $\mathrm{S}$, Tulsky $\mathrm{D}$ et al. Acupuncture for chronic shoulder pain in persons with spinal cord injury: a small-scale clinical trial. Arch Phys Med Rehabil 2007; 88: 1276-1283.

38 Wong AM, Leong CP, Su TY, Yu SW, Tsai WC, Chen CP. Clinical trial of acupuncture for patients with spinal cord injuries. Am J Phys Med Rehabil 2003; 82: 21-27.

39 Curtis KA, Tyner TM, Zachary L, Lentell G, Brink D, Didyk T et al. Effect of a standard exercise protocol on shoulder pain in longterm wheelchair users. Spinal Cord 1999; 37: 421-429.

40 Klose KJ, Schmidt DL, Needham BM, Brucker BS, Green BA, Ayyar DR. Rehabilitation therapy for patients with long-term spinal cord injuries. Arch Phys Med Rehabil 1990; 71: 659-662.

41 Klose KJ, Needham BM, Schmidt D, Broton JG, Green BA. An assessment of the contribution of electromyographic biofeedback as an adjunct therapy in the physical training of spinal cord injured persons. Arch Phys Med Rehabil 1993; 74: $453-456$.
42 Lopes P, Figoni SF, Perkash I. Upper limb exercise effect on tilt tolerance during orthostatic training of patients with spinal cord injury. Arch Phys Med Rehabil 1984; 65: 251-253.

43 Warden SJ, Bennell KL, Matthews B, Brown DJ, McMeeken JM, Wark JD. Efficacy of low-intensity pulsed ultrasound in the prevention of osteoporosis following spinal cord injury. Bone 2001; 29: 431-436.

44 Crosby RD, Kolotkin RL, Williams GR. Defining clinically meaningful change in health-related quality of life. J Clin Epidemiol 2003; 56: 395-407.

45 de Vet HC, Ostelo RW, Terwee CB, van der Roer N, Knol DL, Beckerman $\mathrm{H}$ et al. Minimally important change determined by a visual method integrating an anchor-based and a distributionbased approach. Qual Life Res 2007; 16: 131-142.

46 Wade DT. Outcome measures for clinical rehabilitation trials: impairment, function, quality of life, or value? Am J Phys Med Rehabil 2003; 82 (suppl): S26-S31.

47 Marino RJ. Domains of outcomes in spinal cord injury for clinical trials to improve neurological function. J Rehabil Res Dev 2007; 44: 113-121.

48 Herbert R, Jamtvedt G, Mead J, Hagen K. Practical Evidence-Based Physiotherapy. Elsevier: Oxford, 2005.

49 Schulz KF, Chalmers I, Hayes RJ, Altman DG. Empirical evidence of bias. Dimensions of methodological quality associated with estimates of treatment effects in controlled trials. JAMA 1995; 273: 408-412.

50 Moher D, Pham B, Jones A, Cook DJ, Jadad AR, Hoer M et al. Does quality of reports of randomised trials affect estimates of intervention efficacy reported in meta-analyses? Lancet 1998; 352: 609-613.

51 Siersma V, Als-Nielsen B, Chen W, Hilden J, Gluud L, Gluud C. Multivariable modelling for meta-epidemiological assessment of the association between trial quality and treatment effects estimated in randomized clinical trials. Stat Med 2007; 26: 2745-2758.

52 Gluud L. Bias in clinical intervention research. Am J Epidemiol 2006; 163: 493-501. 